\section{(6) OPEN ACCESS}

\title{
Prospective study of sentinel lymph node biopsy for conjunctival melanoma
}

\author{
Victoria M L Cohen, ${ }^{1}$ Maria Tsimpida, ${ }^{1}$ John L Hungerford, ${ }^{1}$ Hikmat Jan, ${ }^{2}$ Rino Cerio, ${ }^{3}$ \\ Graeme Moir $^{4}$
}

${ }^{1}$ Department of Ocular Oncology, St Bartholomew's Hospital and Moorfields Eye Hospital London, London, UK ${ }^{2}$ Department of Nuclear Medicine, Barts Health NHS Trust, London, UK

${ }^{3}$ Department of Dermatology, Barts Health NHS Trust, London, UK

${ }^{4}$ Department of Plastic Surgery, Barts Health NHS Trust, London, UK

\section{Correspondence to} Victoria M L Cohen, Ophthalmology Department, St Bartholomew's Hospital, West Smithfield

London EC1A 7BE, UK; victoria.cohen@bartshealth. nhs.uk

Received 14 May 2013 Revised 7 August 2013 Accepted 17 August 2013 Published Online First 24 September 2013

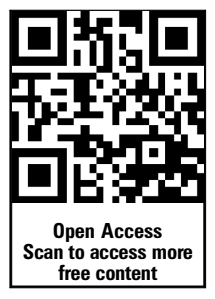

To cite: Cohen VML Tsimpida M, Hungerford JL, et al. Br J Ophthalmol 2013;97:1525-1529.

\section{ABSTRACT \\ Background To report our experience with sentinel} lymph node biopsy for staging patients with conjunctival melanoma.

Methods A prospective review of patients with conjunctival melanoma who underwent sentinel lymph node biopsy at St Bartholomew's Hospital from May 2008 to May 2012. The selection criterion for sentinel node biopsy depended on the tumour thickness $(\geq 2 \mathrm{~mm}$ ) and location of the conjunctival melanoma. The main outcome measures were the incidence of sentinel lymph node positivity and the procedure-related complications.

Results In 4 years, 26 out of 70 patients met the selection criteria for sentinel lymph node biopsy. 4 patients declined and 22 patients consented for the procedure. Technetium-99m failed to identify a sentinel lymph node in four of the 22 patients (18\%). Of the remaining 18 patients, two were found to have subclinical micrometastasis in regional lymph nodes. Median follow-up was 20 months (range 6-36 months). No false-negative events were observed. Complications of the procedure included transient blue staining of the epibulbar surface in five patients and transient facial nerve palsy in one patient.

Conclusions Sentinel lymph node biopsy is a safe procedure with minimal complications. It should be considered for the staging of conjunctival melanomas, especially melanomas in non-limbal location or conjunctival melanomas $\geq 2 \mathrm{~mm}$ thick.

\section{INTRODUCTION}

Conjunctival melanoma is far less prevalent than cutaneous melanoma, with an annual incidence of approximately 0.5 cases per million population. ${ }^{1}$ Conjunctival melanoma can arise from a preexisting naevus, de novo, but is most commonly seen in the setting of primary acquired melanosis (PAM). The incidence has increased over the past 30 years. ${ }^{1}$ Although rare, this ocular surface malignancy is potentially lethal with an average 10-year mortality rate of $30 \% .^{2-4}$

Metastatic spread of conjunctival melanoma can occur via lymphatic drainage to regional lymph node basins or haematogenously to distant organs. The regional lymph nodes for conjunctival melanoma are found in the cervical lymph node chain and parotid gland. The risk of regional lymph node metastasis varies from $20 \%$ to $40 \% .^{5}$ Metastases are rarely found at diagnosis but appear typically at 1-2 years after diagnosis in approximately $10 \%$ of patients. $^{3}$ 5-8 Reported risk factors for regional metastatic spread include primary conjunctival melanoma in non-limbal location, tumour thickness
$>2 \mathrm{~mm}$, large basal diameter, positive resection margins, orbital extension and nodular tumour shape. ${ }^{3}$ Most recently, de novo conjunctival melanoma has been reported to have a higher risk of metastasic spread compared with conjunctival melanoma arising from a pre-existing naevus or from PAM. ${ }^{9}$ Distant metastasis can occur in the brain, lung, liver, gastrointestinal tract, skin and bone. Distant metastatic spread is less common as it is reported in 9\%-25\% of patients. ${ }^{3} 8{ }^{8} 10 \quad 11$ Risk factors for distant metastases include an unfavourable tumour location such as the palpebral conjunctiva, fornices, plica, caruncle and lid margins, tumour thickness greater than $2 \mathrm{~mm}$ and a history of local recurrence. ${ }^{3} 7812$

Regional lymph nodes are known to be the first site of metastasis for the majority of patients with conjunctival melanoma and distant metastases are usually detected later. ${ }^{5}$ Regional metastasis in patients with conjunctival melanoma can be clinically identified by palpation of the regional lymph nodes or can be detected with high resolution ultrasound and MRI of the head and neck. During the last decade, sentinel lymph node biopsy (SLNB) for conjunctival melanoma has allowed the diagnosis of subclinical micrometastasis in regional lymph nodes, enabling us to detect a much smaller disease volume compared with other modalities such as CT or MRI. ${ }^{13-18}$ This provides an early opportunity to treat the cervical lymph node chains before distant metastasis can develop. However, it is important to keep in mind that up to $25 \%$ of patients with metastatic conjunctival melanoma are reported to have distant metastases without clinically detectable regional lymph node involvement. ${ }^{10}$

In this report, we present our experience in 18 patients with conjunctival melanoma who underwent SLNB over a 4-year period. The main outcome measures were the incidence of sentinel lymph node (SLN) positivity and the procedure-related complications.

\section{MATERIALS AND METHODS}

A prospective study of all patients with conjunctival melanoma who underwent SLNB at $\mathrm{St}$ Bartholomew's Hospital from May 2008 to May 2012 was undertaken. The primary selection criterion for SLNB was conjunctival melanoma of two or more millimetre thickness, as determined by histological reporting. The $2 \mathrm{~mm}$ cut-off was selected as this is the thickness reported as a risk factor for regional lymph node metastases in conjunctival melanoma. ${ }^{19}$ Missotten et $a l^{20}$ reported that a thickness of 1-2 mm was associated with a 2.4-fold risk of regional metastases compared with 
$1 \mathrm{~mm}$ or less. The secondary selection criteria included conjunctival melanoma in unfavourable locations (eg, forniceal, caruncular or tarsal conjunctiva) and recurrent conjunctival melanoma associated with the 'florid phase' of PAM. We use florid phase to describe patients who after years of PAM with a history of treated melanoma remission develop multiple new site melanomas over a 1-2-year period. Sentinel node biopsy was particularly useful to stage those scheduled for exenteration. These cases typically have large or recurrent tumours that cannot be controlled with globe sparing surgery. Prior to the procedure, all patients underwent staging for regional and systemic metastases with a full body [18F]-fluorodeoxyglucose positron emission tomography-CT chest and high-resolution neck ultrasound. Only patients with negative metastatic staging were selected for SLNB. Informed consent was written and obtained after discussion of the potential benefits and complications of the procedure. This study conformed to the principles set out in the Declaration of Helsinki.

Melanoma SLN identification by imaging and $\gamma$-probe localisation was performed within 6 weeks of primary excision of the conjunctival melanoma. Any adjuvant treatment required such as cryotherapy or wider local excision was performed at the time of sentinel node biopsy. An amount of $0.1-0.2 \mathrm{~mL}$ of $10 \mathrm{MBq}$ technetium Tc-99m nanocolloid was injected subconjunctivally into the remaining scar (VMLC). Immediately after the injection, dynamic imaging of the ipsilateral head and neck was performed for $10 \mathrm{~min}$, followed by a static image at $30 \mathrm{~min}$, until the first SLN was detected. Transmission images of the head and neck were obtained thereafter. The location of the radioactivity was confirmed with the $\gamma$-probe. The skin overlying the area of high radioactivity was marked with a durable marker pen. An intraoperative $0.2 \mathrm{~mL}$ injection of methylene blue was given into the conjunctival scar to aid in the identification of the SLNs (VMLC). Following the skin incision, sterile handheld $\gamma$-probe was used throughout to localise the SLN (figure 1). Once identified, the SLNs were carefully dissected from a blood free surgical field (GM) and sent for histological evaluation. SLNs are serially sectioned using the 'bread loaf' approach in 2-mm sections; the same pathologist (RC) always examined the H\&E-stained sections for malignant cells. If no malignant cells are detected then immunohistochemical analysis for malignant melanoma is performed using S100 protein, HMB45 and Melan A for every node.

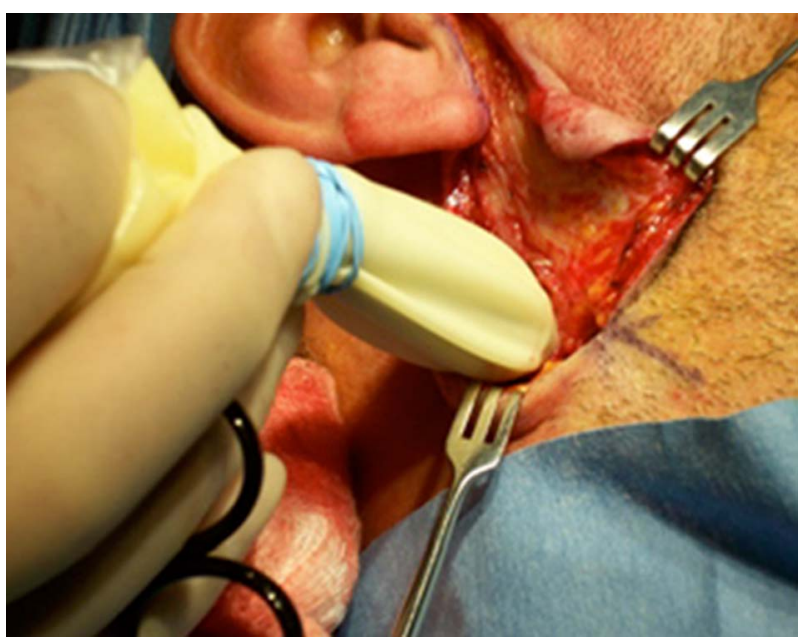

Figure 1 Handheld $\gamma$-probe used to localise the sentinel lymph node.
Information regarding age, sex, predisposing diagnosis, location of tumour, thickness, treatment modality used, location of sentinel nodes, incidence of SLN positivity, procedure-related complications and length of follow-up was collected prospectively.

\section{RESULTS}

A total of 26 out of 70 patients with conjunctival melanoma met the selection criteria for SLNB. Four patients declined SLNB and 22 patients were enrolled in the study. The patients who declined SLNB did not differ from those who consented and have not to date developed metastases. The demographic characteristics of the 22 patients and their tumour features are described in table 1 . In order to determine the tumour thickness and select the patients eligible for SLNB, excisional conjunctival biopsies were performed on a separate day, prior to lymphoscintigraphy (figure 2). Six patients had their incisional/ excisional conjunctival biopsies in outside referring institutions. At St Bartholomew's Hospital, 19 excisional conjunctival biopsies were performed and this included four patients who had had primary incisional surgery elsewhere. Adjuvant cryotherapy was performed in 12 patients, $\beta$-radiotherapy (strontium 90) was applied in six patients and MMC $0.04 \%$ in one patient. On the day of SLNB, prior to the procedure, five patients had adjuvant cryotherapy in the area of the previously excised conjunctival lesion and four patients had wider conjunctival excisions combined with cryotherapy. Of the 22 patients enrolled in the SLN protocol, six patients had a history of recurrent conjunctival melanoma and three had advanced melanoma scheduled for exenteration.

The first patient who underwent SLNB in our hospital was a 12-year-old child diagnosed with malignant transformation of a naevus in the plica, a condition extremely rare in this age group. The conjunctival tumour was managed by a further wide local excision and adjuvant cryotherapy. A preauricular SLN was removed that was negative for micrometastatic disease. The patient continues to be screened in the ocular oncology service.

Table 1 Demographics and tumour characteristics

\begin{tabular}{ll}
\hline & Number or ratio \\
\hline $\begin{array}{l}\text { Demographics } \\
\text { Median age (years) }\end{array}$ & 59 (range: $12-78$ years) \\
Sex & M/F=11/11 \\
Laterality & RE/LE=9/13 \\
Predisposing diagnosis & \\
PAM with atypia & $16(73 \%)$ \\
Naevus & $2(9 \%)$ \\
De novo & $4(18 \%)$ \\
Median duration of PAM (years) & $13($ range $2-30$ years) \\
Tumour characteristics & \\
Location & \\
Bulbar conjunctiva & $7(32 \%)$ \\
Limbal conjunctiva & $6(27 \%)$ \\
Tarsal conjunctiva & $2(9 \%)$ \\
Fornix & $3(14 \%)$ \\
Plica & $2(9 \%)$ \\
Caruncle & $2(9 \%)$ \\
Median tumour thickness (mm) & 2 (range 2-13 mm) \\
& Not stated in 3 patients \\
\hline F, female; LE, left eye; M, male; PAM, primary acquired melanosis; RE, right eye. &
\end{tabular}


Figure 2 Conjunctival melanoma before and after excisional biopsy.
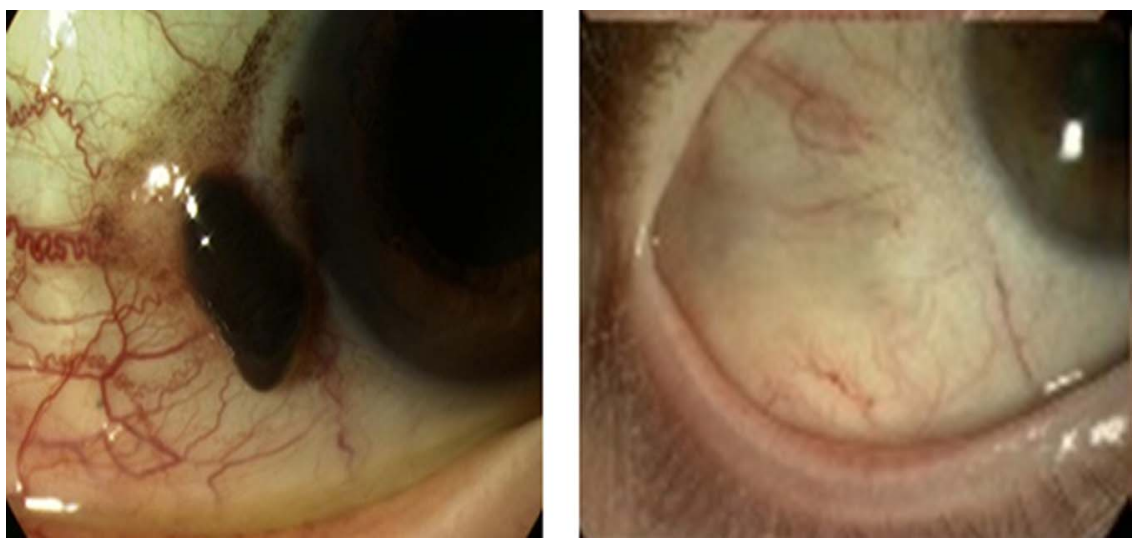

Three years following this procedure, the patient still has no evidence of distant or regional metastasis.

Technetium-99m, with the use of the $\gamma$-probe and $\gamma$-camera, failed to identify the regional lymph nodes in four of the 22 patients (18\%). In the remaining 18 patients, $1-5$ lymph nodes $($ median $=2)$ per patient were identified and removed. The patterns of lymphatic drainage are presented in table 2 .

Only $2(11 \%)$ of 18 patients were found to have positive micrometastatic disease in the SLN. The first patient with histologically positive SLN initially had a $2.2 \mathrm{~mm}$ thick inferior tarsal melanoma associated with PAM with atypia. The patient had undergone a full thickness wedge incisional biopsy of the lower lid in another centre. When he presented to us, he clearly had melanoma infiltrating the anterior lamellar of the lower lid and therefore exenteration was planned to prevent further local spread. He was found to have a positive preauricular node and underwent exenteration with superficial parotidectomy and radical dissection of the lymph node chain. Three years following this procedure he is still alive and disease-free. The second patient had a $10 \mathrm{~mm}$ thick recurrent conjunctival melanoma in the superior fornix associated with PAM with atypia and recurrent melanoma of 10 years duration. At the time of SLNB, a preauricular node was found to be positive. The patient was too medically frail to consider a radical neck dissection so following exenteration surgery she underwent palliative regional radiotherapy to the neck. Three months later she developed clinically palpable cervical lymph nodes, along with a malignant pleural effusion and died. In summary, the two patients with a positive SLN had a history of PAM with atypia. One had undergone an

Table 2 SLNB results in 18 patients

\begin{tabular}{ll}
\hline & Ratio (\%) \\
\hline $\begin{array}{l}\text { Type of lymph nodes } \\
\text { Preauricular }\end{array}$ & $22 / 30(73 \%)$ \\
Jugulodigastric & $4 / 30(13 \%)$ \\
Submandibular & $4 / 30(13 \%)$ \\
Positive lymph nodes & $2 / 18(11 \%)$ of patients \\
Blue nodes & $10 / 30(33 \%)$ \\
Complications & \\
Transient blue stain & $5 / 16(31 \%)$ \\
Transient facial nerve palsy & $1 / 18(6 \%)$ \\
Median follow-up period (months) & $20($ range $6-36$ months) \\
False-negative results & $0 \%$ \\
\hline SLNB, sentinel lymph node biopsy. &
\end{tabular}

incisional biopsy elsewhere for a primary conjunctival melanoma and the other had a recurrent melanoma.

Methylene blue dye was injected subconjunctivally in 16 of the 18 patients. However, only 10 of the 30 (33\%) lymph nodes excised were found to be blue. Residual blue staining of the conjunctival surface was observed in five of the 16 patients (31\%) and persisted for up to 12 months (figure 3). One patient developed an ipsilateral, transient facial nerve palsy following the SLNB procedure, predominantly affecting the frontalis and orbicularis muscles, which resolved 12 months later. None of our patients developed symptoms consistent with Frey's syndrome.

The median follow-up interval was 20 months (range 636 months). During the period of this study one patient died.

\section{DISCUSSION}

SLNB for conjunctival melanoma was first reported in $2001 .^{13}$ Since then, various reports in the literature have emerged, ${ }^{21}$ the majority of these arising from a single institution in the USA, ${ }^{13-}$ 151718 regarding the use of the procedure for eyelid and conjunctival melanoma. In this series, we report our experience with SLNB in 18 patients with pure conjunctival melanoma. It is important to note that no eyelid tumours were included in this series. In contrast to other ocular oncologists who perform the procedure, we prefer to excise the primary conjunctival lesion on a different day prior to lymphoscintigraphy and SLNB. This gives us the opportunity to measure the tumour thickness and select the patients eligible for the procedure.

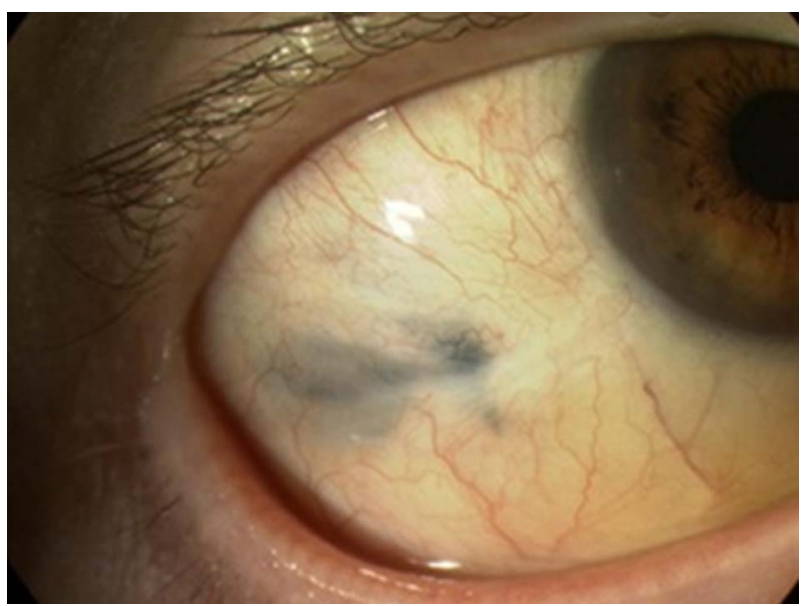

Figure 3 Residual blue staining of the conjunctival surface. 
Moreover, by injecting the Technetium-99m nanocolloid in the area of the previously excised conjunctival melanoma, we minimise the potential risk of tumour seeding under the loosely attached conjunctival surface to a distant ocular site such as a rectus muscle.

In this series, we report four cases (18\%) where the radioisotope failed to localise the SLN; this was termed injection failure. Two of the four patients with injection failure had multiple surgeries for recurrent conjunctival melanoma and one had previous $\beta$-radiotherapy to the conjunctiva. Therefore, in our experience, injection failure may occur when there is conjunctival scarring from surgery or radiotherapy which prevents the technetium reaching the normal lymphatic channels. ${ }^{22}$ The injection failure in one of the cases was seen following exenteration surgery and may have been the result of performing sentinel node biopsy as a secondary procedure. As a result, any patient scheduled for an exenteration will have sentinel node biopsy performed at the time of primary surgery. However, in the majority of cases the injection was successful. Therefore, secondary sentinel node biopsy should not be ruled out if a patient presents with a recent history of excision of a conjunctival melanoma. Patients should be informed of injection failure when consenting for sentinel node biopsy. One may argue that the dose of Tc-99m should be increased to prevent injection failure but there is a risk that with a larger injection volume the radioactivity can spread into areas of lymphatic drainage not representative of the sentinel node draining the primary lesion. Another difficulty experienced with the procedure was leakage of technetium onto the ocular surface and ipsilateral cheek. In order to prevent this complication we now cover the eye directly after the injection and turn the head to the opposite side.

Classic anatomy of the lymphatic drainage patterns from the ocular adnexal region suggests that the temporal lesions tend to metastasise to the preauricular nodes and the nasal lesions to the submandibular and deeper cervical nodes. ${ }^{23}$ Variations in the aforementioned drainage patterns were seen in this series. Two patients with plica lesions, two patients with caruncle lesions and two patients with a lesion in the nasal limbus all showed lymphatic drainage to preauricular nodes instead of submandibular and/or jugulogastric nodes. This variability in drainage patterns was also observed in other reports and highlights the need for further studies. ${ }^{16} 18$

In this series, two of the $18(11 \%)$ patients with conjunctival melanoma who underwent SLNB were found to have regional lymph node micrometastasis. Savar et $a l^{18}$ at MD Anderson in Texas reported an SLN positivity rate of $16 \%$. Their tumour sizes were similar to ours; the melanoma thickness in our SLN positive cases was 2.2 and $10 \mathrm{~mm}$, and by comparison the melanoma thickness in the positive cases reported from the MD Anderson were 2.1, 3.8, 5.2, 6 and $7 \mathrm{~mm}^{18}$ Overall, the median tumour thickness in the patients with a negative sentinel node was $2.0 \mathrm{~mm}$ (range $1.0-13.0 \mathrm{~mm}$ ) in this series compared with $2.7 \mathrm{~mm}$ (range $0.62-12.0 \mathrm{~mm}$ ) in the series from MD Anderson. However, Savar et al included four eyelid melanomas ( 26 conjunctival melanomas), one of which had a positive sentinel node biopsy. Cutaneous melanoma is reported to have a greater risk of regional lymph node micrometastases as cutaneous melanoma greater than $1 \mathrm{~mm}$ Breslow thickness has an SLN positivity rate of $20 \% .{ }^{24}$ This may explain why our SLN positivity rate is lower than that found by the other main centre performing this procedure. Although the rate of SLN positivity is considered to be small, we believe that patients with subclinical nodal metastasis benefit from the procedure as it provides an opportunity to treat micrometastatic disease and increase patient survival. The one patient in our series with a positive SLN who underwent radical neck dissection is still alive 3 years later.

Esmaeli reported that isosulfan dye does not lead to permanent tattooing of the ocular surface and in the majority of cases the colour dissipated over the first $24 \mathrm{~h}$ after injection. ${ }^{14}$ Methylene blue was used in preference over isosulfan blue because methylene blue is frequently used in the London Ocular Oncology Service to mark the surface of the eye with an intraocular melanoma and no residual staining or side effects have been experienced. However, following a $0.1 \mathrm{~mL}$ subcojunctival injection of methylene blue, we observed residual transient staining of the conjunctival surface in five patients that in three patients persisted for up to 12 months. In addition, the blue dye was not particularly helpful in identifying the sentinel node as only 10 of the 30 nodes excised were found to be blue. For the above reasons, we have now abandoned the use of methlyene blue dye, similar to other centres that have been doing SLNBs for ocular tumours. ${ }^{17}$ We are currently only using Tc-99m for the identification of the SLNs.

One of the most serious complications of SLNB in the parotid area is damage to the facial nerve and/or its branches. In this series, we had one case of transient facial nerve palsy $(6 \%)$ that resolved after 12 months. This was the only parotid dissection into the deep lobe. Surgeons should be prepared to abandon SLNB when the dissection goes uncomfortably deep or there is 7 th cranial nerve twitching. Although in the hands of an experienced surgeon this side effect is rare, because of the complex anatomy of the parotid region, all patients should be aware of this side effect prior to consenting for the procedure. It is very important that the anaesthetist does not administer a muscle relaxant as this prevents the surgeon from detecting a facial twitch that indicates imminent damage to the facial nerve. Moreover, the use of bipolar diathermy for dissection can act as a nerve stimulator.

During the period of follow-up following the SLNB procedure, only one patient died of metastatic spread and the remaining 17 patients remained free of regional and/or distant metastasis. The SLNB was positive in the patient who died. Regional radiotherapy failed to control the detected metastatic spread in this patient but she was not well enough to undergo neck dissection. Radiation therapy alone is not considered adequate for the management of a positive SLN basin and only reserved for poor surgical candidates. A large series reviewing the management of patients with positive regional lymph nodes is needed to answer this question.

No false-negative events were observed in this study after a maximum follow-up interval of 3 years. This may be due to the skill of the surgeon (GM) who has vast experience with head and neck dissection. Two reports from the same centre in the USA have reported false-negative event rates of $16 \%$ and $8 \%$, respectively. ${ }^{17} 18$ These were attributed to a surgical learning curve. $^{25}$ Nevertheless, longer follow-up is required and in our unit all conjunctival melanoma patients are screened for evidence of regional and/or distal metastasis at 4-monthly intervals.

To conclude, SLNB is a safe procedure in the hands of an experienced ophthalmic and head and neck or plastic surgeon, with minimal complications. It should be considered for the staging of patients with high risk conjunctival melanoma, especially if found in a non-limbal location or conjunctival melanoma that is $\geq 2 \mathrm{~mm}$ thick.

Contributors VMLC has conceived and designed the study, supervised the research, interpreted data and critically revised the manuscript. She has rewritten the manuscript following review by the BJO. MT collected, analysed and helped draft the 
manuscript. JLH has supervised the research and revised critically the manuscript. $H J$ and GM interpreted data and revised critically the manuscript. RC reported on all the pathology and contributed to the pathology section. All authors have read and approved the final manuscript.

Competing interests None.

Ethics approval The sentinel lymph node biopsy service is established for the staging of melanoma in our hospital.

Patient consent All patients received fully informed consent.

Provenance and peer review Not commissioned; externally peer reviewed.

Open Access This is an Open Access article distributed in accordance with the Creative Commons Attribution Non Commercial (CC BY-NC 3.0) license, which permits others to distribute, remix, adapt, build upon this work non-commercially, and license their derivative works on different terms, provided the original work is properly cited and the use is non-commercial. See: http://creativecommons.org/ licenses/by-nc/3.0/

\section{REFERENCES}

1 Triay E, Bergman L, Nilsson B, et al. Time trends in the incidence of conjunctival melanoma in Sweden. Br J Ophthalmol 2009:93:1524-8.

2 Seregard S. Conjunctival melanoma. Surv Ophthalmol 1998;42:321-50.

3 Shields $\mathrm{CL}$, Shields JA, Gündüz K, et al. Conjunctival melanoma: risk factors for recurrence, exenteration, metastasis, and death in 150 consecutive patients. Arch Ophthalmol 2000;118:1497-507.

4 Brownstein S. Malignant melanoma of the conjunctiva. Cancer Control 2004:11:310-16.

5 Esmaeli B, Wang X, Youssef A, et al. Patterns of regional and distant metastasis in patients with conjunctival melanoma: experience at a cancer center over four decades. Ophthalmology 2001;108:2101-5.

6 Tuomaala S, Eskelin S, Tarkkanen A, et al. Population-based assessment of clinical characteristics predicting outcome of conjunctival melanoma in whites. Invest Ophthalmol Vis Sci 2002;43:3399-408.

7 Paridaens $A D$, Minassian DC, McCartney $A C$, et al. Prognostic factors in primary malignant melanoma of the conjunctiva: a clinicopathological study of 256 cases. Br J Ophthalmol 1994;78:252-9.

8 Missotten GS, Keijser S, De Keizer RJW, et al. Conjunctival melanoma in the Netherlands: a nationwide study. Invest Ophthalmol Vis Sci 2005;46:75-82.

9 Shields CL, Markowitz JS, Belinsky I, et al. Conjunctival melanoma: outcomes based on tumor origin in 382 consecutive cases. Ophthalmology 2011;118:389-95 e1-2.
10 Werschnik C, Lommatzsch PK. Long-term follow-up of patients with conjunctival melanoma. Am J Clin Oncol 2002;25:248-55.

11 Cohen VML, Ahmadi-lari S, Hungerford JL. Gastric metastases from conjunctival melanoma. Melanoma Res 2007;17:255-6.

12 De Potter $\mathrm{P}$, Shields $\mathrm{CL}$, Shields JA, et al. Clinical predictive factors for development of recurrence and metastasis in conjunctival melanoma: a review of 68 cases. $\mathrm{Br} J$ Ophthalmol 1993;77:624-30.

13 Esmaeli B, Eicher S, Popp J, et al. Sentinel lymph node biopsy for conjunctival melanoma. Ophthal Plast Reconstr Surg 2001;17:436-42.

14 Esmaeli B. Sentinel node biopsy as a tool for accurate staging of eyelid and conjunctival malignancies. Curr Opin Ophthalmol 2002;13:317-23.

15 Amato M, Esmaeli B, Ahmadi MA, et al. Feasibility of preoperative lymphoscintigraphy for identification of sentinel lymph nodes in patients with conjunctival and periocular skin malignancies. Ophthal Plast Reconstr Surg 2003;19:102-6.

16 Nijhawan N, Ross MI, Diba R, et al. Experience with sentinel lymph node biopsy for eyelid and conjunctival malignancies at a cancer center. Ophthal Plast Reconstr Surg 2004:20:291-5.

17 Ho VH, Ross MI, Prieto VG, et al. Sentinel lymph node biopsy for sebaceous cell carcinoma and melanoma of the ocular adnexa. Arch Otolaryngol Head Neck Surg 2007:133:820-6.

18 Savar A, Ross MI, Prieto VG, et al. Sentinel lymph node biopsy for ocular adnexal melanoma: experience in 30 patients. Ophthalmology 2009;116:2217-23.

19 Shields CL, Shields JA, Gunduz K, et al. Conjunctival melanoma: risk factors for recurrence, exenteration, metastasis, and death in 150 consecutive patients. Arch Ophthalmol 2000;118:1497-507.

20 Missotten GS, Keijser S, De Keizer RJ, et al. Conjunctival melanoma in the Netherlands: a nationwide study. Invest Ophthalmol Vis Sci 2005;46:75-82.

21 Wilson MW, Fleming JC, Fleming RM, et al. Sentinel node biopsy for orbital and ocular adnexal tumors. Ophthal Plast Reconstr Surg 2001;17:338-44; discussion 44-45-44; discussion 44-45

22 Bilchik AJ, Giuliano A, Essner R, et al. Universal application of intraoperative lymphatic mapping and sentinel lymphadenectomy in solid neoplasms. Cancer J Sci Am 1998:4:351-8.

23 Sherman DD, Gonnering RS, Wallow IH, et al. Identification of orbital lymphatics: enzyme histochemical light microscopic and electron microscopic studies. Ophthal Plast Reconstr Surg 1993;9:153-69.

24 Gomez-Rivera F, Santillan A, McMurphey AB, et al. Sentinel node biopsy in patients with cutaneous melanoma of the head and neck: recurrence and survival study. Head Neck 2008;30:1284-94.

25 Pfeiffer ML, Savar A, Esmaeli B. Sentinel lymph node biopsy for eyelid and conjunctival tumors: what have we learned in the past decade? Ophthal Plast Reconstr Surg 2013;29:57-62. 